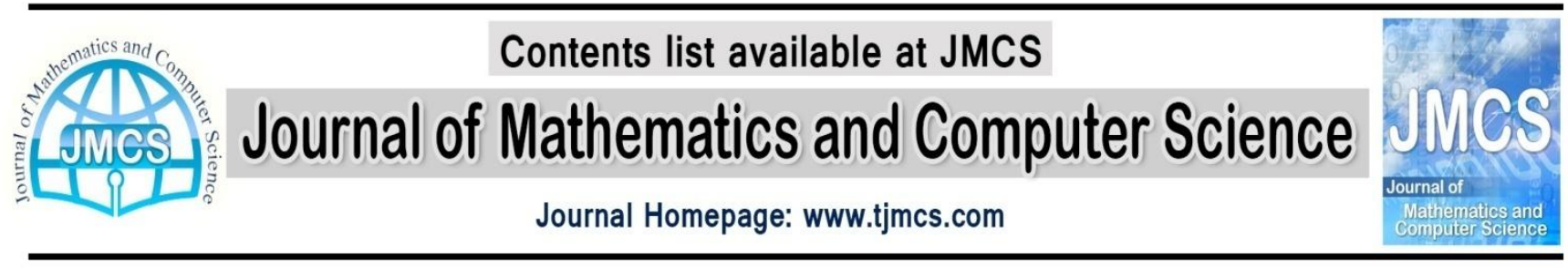

\title{
Dose-Response Modeling applications in field of environmental health
}

\author{
Habib Jafari ${ }^{1}$, Homayoun Sadeghi Bazargani ${ }^{2}$, Yazdan Khaki ${ }^{*}$, Tohid Jafari ${ }^{4}$ \\ ${ }^{I}$ Assistant professor Statistic faculty, Razi University of Kermanshah \\ ${ }^{2}$ Assistant professor Epidemiology and Statistics, Road traffic injury research center, Department of \\ statistics \& epidemiology, Tabriz University of medical science, Tabriz, Iran \\ ${ }^{3}$ M.S. in statistic, Razi University of Kermanshah \\ ${ }^{4}$ Young Researchers and Elite Club, Tabriz Branch, Islamic Azad University, Tabriz, Iran \\ Jafari habib@yahoo.com
}

\begin{abstract}
Article history:
Received December 2013

Accepted January 2014

Available online February 2014
\end{abstract}

\begin{abstract}
Characteristics and applications of benchmark Dose (BMD) modeling in assessing environmental risks affecting the health status is discussed in this article. BMD is a method usually used to estimate the critical dose or the amount of a chemical leading to a known outcome.

This article discusses a statistic model processing Dose-Response variables as a clear and precise answer that introduces constancy and stability and shows that it is a more basic and practical rather than old and traditional method to assess risk of chemicals by assigning their critical volume or Dose.
\end{abstract}

Keywords: Dose-Response, Benchmark Dose (BMD), BMDL, Risk Assessment, U.S.EPA, BMR, LOAEL, NOAEL.

\section{Introduction}

According to ever increasing technology and modernism of our surrounding environment and being exposed to contaminant chemicals either in occupational environment are generally the surrounding areas in having undesirable revenues on the societies sanitation and health condition we have decided to take an action based on statistical modeling. Dose-Response founded on benchmark Dose method to assess the risk and to assign the critical Dose of chemicals in the occupational environment and in a higher level to assign the critical amount in daily consuming product in everyday life deuced by Crump in 1984 is a formulation and methodological way which is used instead of the old and traditional way known as the NOAEL (No -observed -Adverse -Effect- Level) and LOAEL (Lowest -observed-Adverse-Effect- Level) [14]. 
In fact BMD method is established instead of the old traditional way known as LOAEL or NOAEL. Existing limitation for Dose-Response for BMD better appliance [7].

Some criticize that have been brought upon NOAEL method is discussed in below:

-NOAEL way was limited to experimental examination Dose.

-Dose -Response graph and figures wasn't considered.

-Experiments related to animals showed which fewer numbers of animals were inclined to produce larger NOAEL.

-Usually NOAEL is derived from information related to animals and is defined as the highest experimental Dose Level that is considerably different.

-Often NOAEL examined detached Doses in a research.

-NOAEL use didn't collect estimated risk potential.

In poison studies, the NOAEL low poison level is unknown where as in BMD method is well defined [8],[12],[7].

BMD method includes representing observational Dose-Response graph modeling with a large data extent [8]. Model use of this method also states a high accuracy Dose estimation which relates to a unique level of response [12].

What needs to be considered is that BMD method has reduced the statistical accordance that helped an increase in 1 to $10 \%$ in risk. Low boundary level of accordance of 95\% BMD is called BMDL.

The software is designed by the U. S. environmental protecting agency (U.S.EPA) called BMDS in order to assess the risk and to estimate critical chemical Dose release in surrounding environment especially in the occupational environment.

\section{Subject}

One of the goals being considered in this article is the risk assessment process in chemical, in a way that, the world health organization (WHO) has divided the risk assessment process in chemical into four stages:

\section{Risk detection}

2. Dose assessment response

3. Assessment representation

4. Risk features

\subsection{Risk identification:}

Risk detection is a detection of natural abilities because of side effects on health. Usually chemical risk of IN VIVO experiments on animals will be detected. In some cases chemical risk primarily in human epidemiologic studies have been proved.

\subsection{Dose-response assessment:}

Dose-response assessment includes detecting the relation between a chemical Dose and its biological effects. These analyzing also are done on animals, a group of animals are exposed to a given amount of chemicals. And their response to the special treatment will be examined.

\subsection{Exposure assessment:}

Assessment representation that can be done both in qualitative forms includes estimating a degree of human population whom are exposed to the risky chemical.

\subsection{Risk characteristics:}

Risk characteristics are used for the combination and mutual assessment of the risk detecting, Dose assessment response and assessment representation. For example, comparing the output 
condition of Dose assessment response analyzing, by protecting information of the output condition will be gained.

Therefore, it can be Resulted that whether a community or a group is in positional danger or not? Another aim of risk features is detecting the level of the accepted daily consuming for a particular human population [7]

Another aim of this article we have mentioned is a BMD content examination, which includes processing and feting a statistic modeling Dose-Response data, in another word the relation between its Dose amount and biological level. We can mention the estimated critical Dose amount of chemicals and contaminant that the worker is exposed to in the occupational environment and also to mention another aim is to study suitable and preventable procedures of undesirable side effects unhealthy condition of these people in such places.

\section{Methods}

According it, this article which is a diagnostically study and major practice of BMD as a Dose, has caused undesirable effects on sanitation and health conditions compared to the past, we have decided to introduce different models for both quanta and continuous variables to make a maximum diffraction undesirable health and sanitation effect improvement in our environment [7].

The set of data for calculating BMD should have the following conditions:

1-There must be at least one considerable statistical or biological process in accordance to the Dose of the end points.

2-The set of data that is used for modeling should include information related Dose-Response [12]. As its been mentioned above, the aim of model processing is a way to find the model parameters amount, so that can describe the result of model processing. In fact the process starts with a guess for parameter amount [9].

These guesses are updated frequently to make a continuation that becomes convergent several ways have been represented to converge correct parameters for a set of data to gain each proportional set of primary parameters amount.

It needs to be considered the present procedures for cancer risk assessment by U.S environmental protection agency (U.S.EPA) shows no priority for one particular model, but suggests some of DoseResponse models that are used for estimating the lower limits of Dose related to risk levels of 1, 5 and $10 \%$ [7].

Dose -Response models for quantal data some models for risk assessment for quantal data have been suggested in chart 1 :

Chart 1- Dose-Response models for quantal data

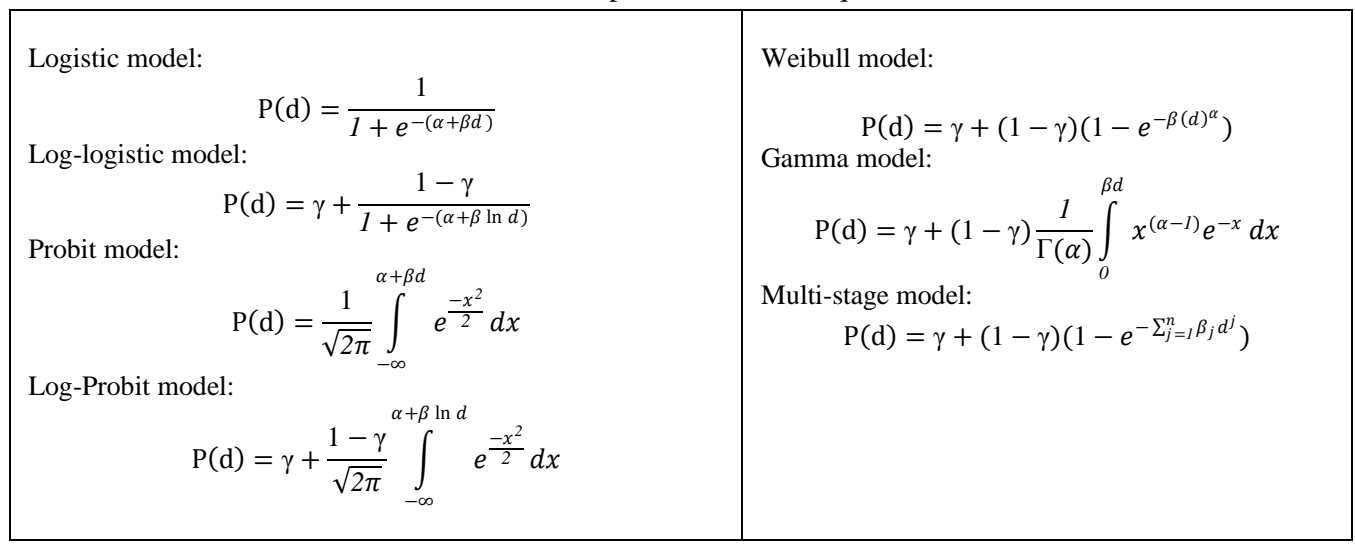


The first experiment and researches were on quantitative response variable based on litter that was analyzed by Weibull model. Parallel to these two different quantitative definitions has been represented by benchmark response level (BMR).

BMR might be defined in two forms: In which $\mathbf{p}(\mathbf{B M D})$ is the chance of response in $B M D$ and $\mathbf{p}(\mathbf{0})$ is the chance of the pre-response.

These two definitions are equal if pre-data are zero. Extra risk was used more than additional risk, which its reason was because of having an antecedent and $10 \%$ level used in dedication that is a considerable difference [7].

Chart 2- statistic models for continuous data

\begin{tabular}{|c|c|}
\hline Dose-Response models & Variance models \\
\hline $\begin{array}{l}\text { Polynomial model: } \\
\qquad \mu(d)=\alpha+\beta_{1} d^{l}+\cdots+\beta_{n} d^{n} \\
\text { Power model: } \\
\qquad \mu(d)=\alpha+\beta d^{\eta} \\
\text { Hill function: } \\
\qquad \mu(d)=\alpha+\theta \frac{d^{\eta}}{k^{\eta}+d^{\eta}} \\
\text { Richards function: } \\
\qquad \mu(d)=\alpha+\theta\left[\frac{d^{\eta}}{k^{\eta}+d^{\eta}}\right]^{\nu} \\
\text { Exponential model: } \\
\qquad(d)=\alpha+\theta\left(1-e^{-\left(\frac{d^{\eta}}{k^{\eta}}\right)}\right)\end{array}$ & $\begin{array}{l}\text { Constant model: } \\
\qquad \sigma^{2}(d)=C \\
\text { Powerful function of men: } \\
\qquad \sigma^{2}(d)=\lambda[\mu(d)]^{\rho} \\
\text { Dose dependent exponential model: } \\
\qquad \sigma^{2}(d)=e^{\lambda+\rho \ln (d+1)}\end{array}$ \\
\hline
\end{tabular}

Statistical models have been represented for connecting data also in chart 2.

We need to know many models have been used to describe the relation between Dose or chemical volume with an average response of $\boldsymbol{\mu}$ (d) that two models named Polynomial and Power model have been used in the past in particular modeling practices in lower connected spots [7]. Chart2 also, includes other models such as hill function, that this chance was usually used in DoseResponse models in pharmacology [7].

For several practical reasons about chemical risk assessment and BMD is analyzing method and estimate of critical Dose spots by hills function, a generalization was introduced by Richards named Richards function.

As it is shown in chart 2, some models of variance might be regarded too.

Although it's better to assume a variance for each fixed Dose but it's not suitable to do so for biological data and when response average increases, the variance increases and vice versa [9].

According to represented models, some popular ways in general are used for model procession:

1- Nonlinear least squares method 


\section{2-Maximum likelihood method}

\section{3-Generalization equivalent estimation (GEE) method}

\subsection{Nonlinear least squares method}

Least non-matrix squares method is a popular method in connecting variables is used when the observations are considered independently. The base assumption is that the observational variance of the average surrounding group dose is fixed among Doses [1].

The last non-matrix squares method includes different squares set between observed data and predicted amounts. This method is suitable particularly in biological discussion, when the data could have at least in an approximate level normal attribution.

\subsection{Maximum likelihood method:}

Maximum likelihood method is a general method for extracting desirable function when we could make a suitable theory about data attribution because these estimates by maximum likelihood method have suitable statistical properties. Maximum likelihood method is a preferable form of estimates for the time that logical assumption is closer to reality.

\subsection{Generalization equivalent estimation (GEE):}

The third group of the parameter estimate method is related to semi chance method. In GEE it is necessary to detect the average, variance and structural correlation. GEE method is similar to estimates of likelihood function which is used for depended observation (like litter) [9],[7].

\section{Conclusion}

The use of some evident examples has been shown that the definition and detection of BMD and BMDL depends on model choosing. When different models can have different results in low Dose spots, then it will become discussing [10].

We can mention some examples of the side effects and diseases caused by being exposed to chemicals and contaminant materials in environment for workers and people who are around:

-Estimating the suffering risk of kidney diseases (ESRD) that is caused by being exposed to Aristolochec Asid [13].

-Audition lateness of brain stem finally causes in postponing the children's potential activities and also a difference in being vulnerable to poisoning of being exposed to mercury methyl [8].

-Critical Dose estimate influential on balance and disorder in neurological system activities in Japan, caused by being exposed to lead [2].

-Assessment of standard Dose (BMD) for bone cavity in China caused by being to cadmium [6].

-The estimated standard Dose for bone damage and skeleton system in individual and kidney disorders in Chinese men in their occupational environment caused by being exposed to lead [11].

-Chromosome damage assessment for workers by BMD in central China caused by being exposed vinyl chloride [3].

-Risk assessment, neuron guiding speed in workers because of lead [16].

-BMD is used in human liver insufficiency studies because of arsenic produced by burning coal [15]. The jobs involving chemicals like: lead, cadmium, arsenic and other, according to the observations, workers working with these chemicals need more protection especially female workers who are most vulnerable [10].

As the old and traditional NOAEL cannot provide potential risk (one of its limitations) BMD method has caused an increase of 1 to $10 \%$ of risk taking level by reducing statistic assurance gap. In other words it is shown that researchers and scientists tend to have results with short assurance gap and thus lower assurance. 


\section{References}

[1] Khorsi, M., Bozorgi-Amiri, A. and Ashjari, B. (2013). A Nonlinear Dynamic Logistics Model for Disaster Response under Uncertainty. The Journal of Mathematics and computer Science, Vol. 7, Issue 1, pp. 63-72.

[2] Iwata, T. Yano, E. Karita, K. Dakeishi, M. Murata, K. (2005). Critical dose of lead affecting postural balance in workers. American Journal of Industrial Medicine. Vol. 48, Issue 5,pp. 319-325.

[3] Jiao, J. Feng, N.-N. Li, Y. Sun, Y.Yao, W. Wang, W. Zhang, G.-H.Sun, S.-Y. Tan, H.-S. Wang, Q. Zhu, Y. Li, Y. Brandt-Rauf, P.W.Xia, Z.-L. (2012). Estimation of a safe level for occupational exposure to vinyl chloride using a benchmark dose method in central china. Journal of Occupational Health. Vol.54, Issue 4,pp. 263-270

[4] Murata, K., Weihe, P., Budtz-Jørgensen, E., Jørgensen, P.J., Grandjean, P. (2004). Delayed brainstem auditory evoked potential latencies in 14-year-old children exposed to methyl mercury. Journal of Pediatrics . Vol. 144, Issue 2,pp. 177-183.

[5] Nazif, H. (2012). A Genetic Algorithm for Solving Scheduling Problem. The Journal of Mathematics and computer Science, Vol.5, Issue 2, pp. 91-96.

[6]. Qian, H.L., Jin, T.Y., Kong, Q.H., Wang, H.F., Zhu, G.Y. (2006). Application of benchmark dose (BMD) in a bone-effect study on a general population environmentally exposed to cadmium. Chinese journal of industrial hygiene and occupational diseases. Vol. 24, Issue 1, pp. 23-26

[7] Salomon, S. (2005). Dose-Response Modeling. Evaluation, Application and Development of Procedures for Benchmark Dose Analysis in Health risk Assessment of chemical. Stockholm, Sweden. Thesis.

[8] Salomon J. Sand, Dietrich von R. and Agneta F.F. (2003). Benchmark Calculations In Risk Assessment Using Continuous Dose-Response Information: The Influence of Variance and the Determination of a Cut-Off Value. Risk Analysis, Vol. 23, No. 5.

[9] Salomon S., Agneta F. F. and Katarina V. (2002). Evaluation of the Benchmark Dose Method for Dichotomous Data: Model Dependence and Model Selection. Regulatory Toxicology and Pharmacology 36, 184-197. doi:10.1006/rtph.2002.1578.

[10] Sakuragi, S. , Takahashi, K. Hoshuyama, T. Moriguchi, J. Ohashi, F. Fukui, Y. Ikeda, M. （2012). Variation in benchmark dose (BMD) and the 95\% lower confidence limit of benchmark dose (BMDL) among general Japanese populations with no anthropogenic exposure to cadmium. International Archives of Occupational and Environmental Health Vol. 85, Issue 8, pp. 941-950.

[11] Sun, Y. Sun, D.Zhou, Z.Zhu, G. Lei, L. Zhang, H. Chang, X. Jin, T. (2008). Estimation of Benchmark dose for bone damage and renal dysfunction in a Chinese male population occupationally exposed to lead. Annals of Occupational Hygiene. Vol.52, Issue 6, pp. 527-533.

[12] WoodrowSetzer, Jr. and Carole A. Kimmel (2003). Use of NOAEL, benchmark dose, and other models for human risk assessment of hormonally active substances. Pure Appl. Chem., Vol.75, 
Nos. 11-12, pp. 2151-2158.

[13] Wu, F.Wang, T. Risk assessment of upper tracturothelial carcinoma related to aristolochic Acid (2013). Cancer Epidemiology Biomarkers and Prevention. Vol. 22, Issue 5,pp. 812-820

[14] Zeng, Q.B. , Yu, X., Yang, J., Hong, F. (2012). Biological exposure limit in bone metabolism damage induced by co-exposure to fluorine and arsenic from coal burning. Chinese Journal of Endemiology. Vol. 31, Issue 5, pp. 523-525

[15] Zhang, A.-H., Li, J., Hong, F., Luo, P., Yang, G.-H., Yang, D.-P., Huang, X.-X., Zhang, B.X., Dong, X.-X. (2009). Application of benchmark dose on the study of people's liver dysfunction induced by arsenic-coal burning and its significance. Chinese Journal of Endemiology. Vol. 28, Issue 2, pp. 157-161

[16] Zheng, G., Lei, L.J., Li, W.H., Chang, X.L., Jin, T.Y., Zhou, Z.J., Tian, L.T., Li, H.Q., Pan, X.H. (2010). Risk assessment of nerve conduction velocity in workers exposed to lead]. Chinese journal of industrial hygiene and occupational diseases. Vol. 28, Issue 3,pp. 164-169. 